\title{
CESTODES DU GENRE RAILLIETINA TROUVÉS CHEZ L'HOMME EN AMÉRIQUE INTERTROPICALE
}

\author{
Par Robert-Ph. DOLLFUS \\ (suile et fin)
}

\section{Les Raillietina de L'homme a Cuba}

La comparaison des Raillietina de l'Equateur ci-dessus décrits (1), avec les espèces de Raillietina connues chez l'homme et les mammifères m'ayant conduit à ne rapporter aucune des premières à l'une des autres; j'ai cherché des formes de comparaison parmi les Raillietina des Antilles. J'étais informé de l'existence de la raillietinose humaine aux Antilles par un document manuscrit conservé à l'Institut de Parasitologie de la Faculté de Médecine de Paris et par une récente publication de P. Kourí et J. M. Doval (1938).

Le document manuscrit était une lettre, datée du 5-4-1930, adressée au professeur E. Brumpt, par les $\mathrm{D}^{\text {rs }}$ R. Hernando Loeches et Pablo Alavez, relatant un cas de raillietinose à Agramonte (Province de Matanzas, Cuba), observé chez un enfant de 16 mois ; un ver avait été trouvé dans les selles et envoyé au prof ${ }^{r} \mathrm{E}$. Brumpt; je l'ai retrouvé dans les collections du laboratoire et le prof ${ }^{r} \mathbf{E}$. Brumpt m'a permis de l'examiner, ce dont je le remercie vivement. L'exemplaire paraît complet, comportant le scolex et le strobile jusqu'aux proglottis gravides à capsules prètes à être libérées. Bien malheureusement, ce ver était vraisemblablement mort depuis quelque temps lorsqu'il a été expulsé par le malade ou bien a èté trop tardivement mis en alcool, car ses tissus sont mal conservés, en dégénérescence, avec noyaux pycnotiques ; il est cependant possible d'en donner une description permettant sa comparaison avec les autres Raillietina.

(1) Tout le matériel (Raillietina) envoyé en communication par le $\mathrm{D}^{\mathrm{r}} \mathrm{L}$. A. León lui a été renvoyé à Quito, à l'exception de : $1^{\circ}$ quelques fragments de strobile prélevés dans chaque tube et utilisés pour des préparations; $2^{\circ}$ les deux préparations de scolex de quitensis, qui ont été provisoirement conservées à l'Institut de Parasitologie de la Faculté de Médecine de Paris.

Annales de Parasitologie, T. XVII, No $6 .-1939-1940$, p. 542-562. 


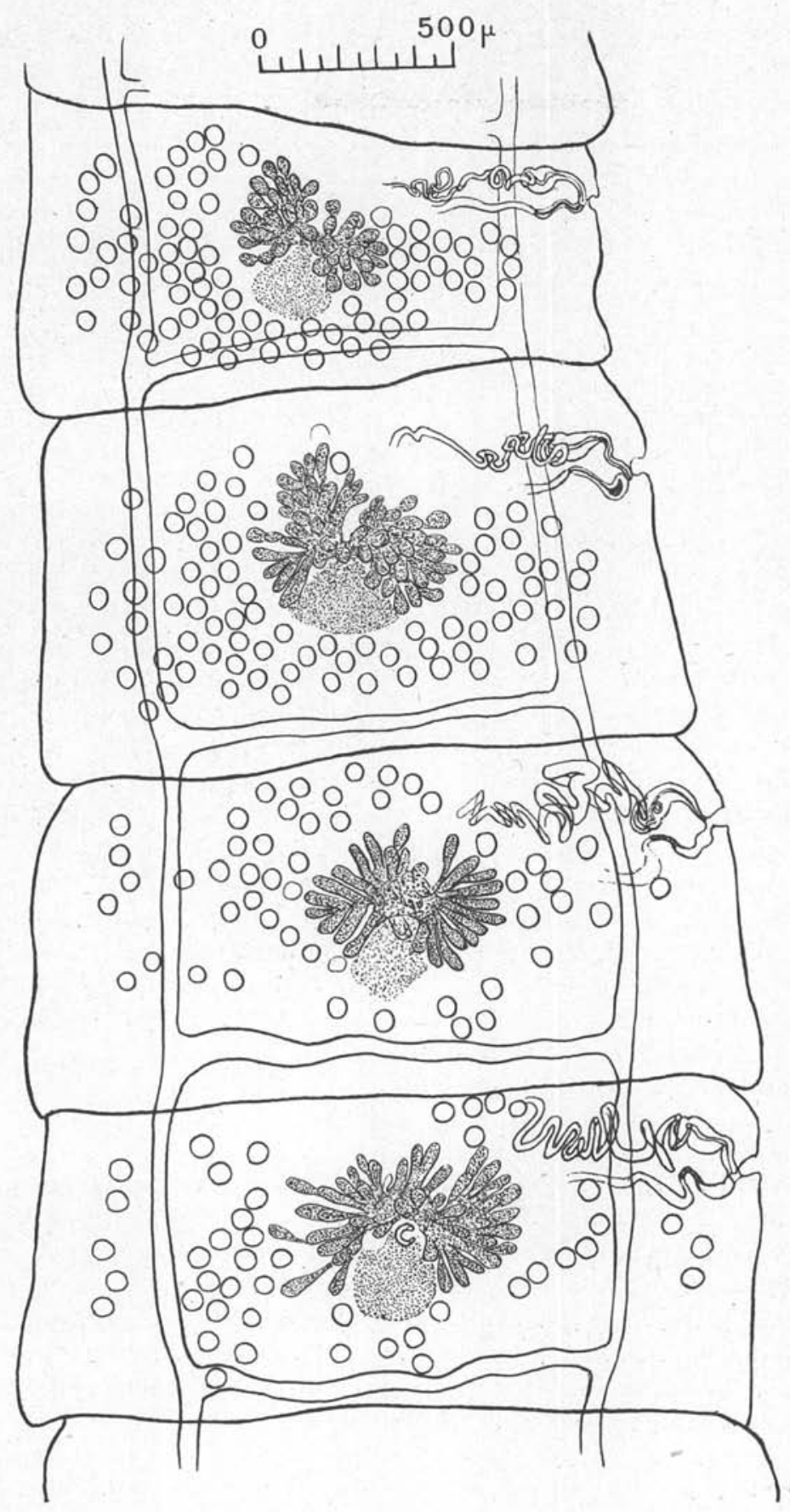

Fig, 21. - R. (R.) equatoriensis R.-Ph. Dollfus 1939. Proglottis sexués montrant la séparation des lobes ovariens en deux éventails. 
C'est un ver plutôt petit (191 mm. de long) et grêle, avec 265 proglottis, bien loin de rappeler l'habitus des grands Raillietina de L. A. León. Le scolex a une largeur d'env. $0 \mathrm{~mm}$., 35, avec des ventouses d'env. 0,148 de diam., il n'y a pas de rostre ni de crochets observables (il est probable que les crochets du rostre et ceux des ventouses, s'il y en avait, étant très caducs, sont tombés). Le strobile a d'abord une

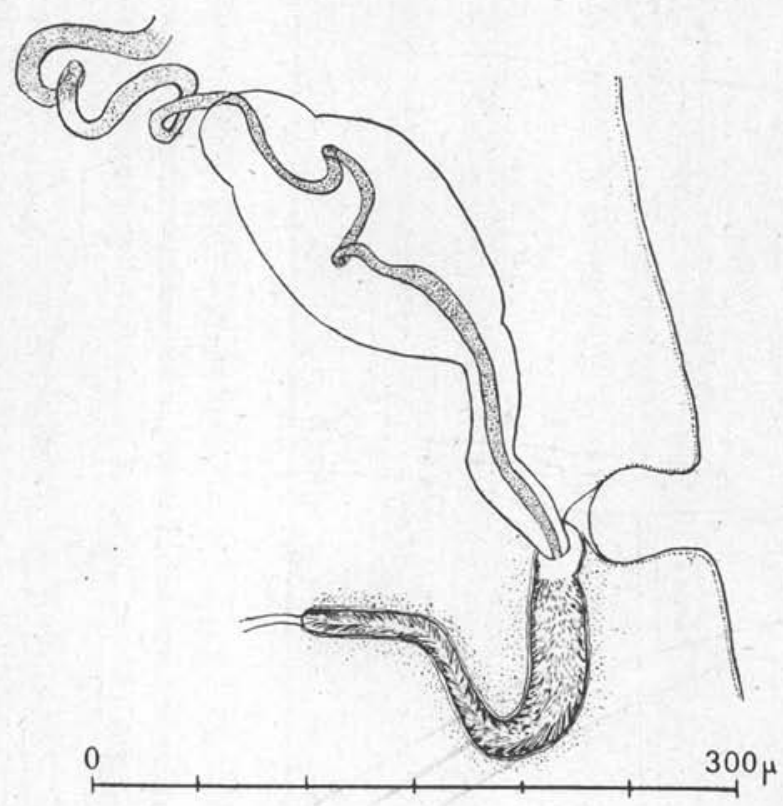

Fig. 22. - R. (R.) equatoriensis R.-Ph. Dollfus 1939. Poche du cirre et vagin. Autofécondation.

largeur d'env. 0,266, puis 0,252, qui augmente insensiblement pour atteindre 0,322 à une distance de $5 \mathrm{~mm}$. du scolex. Les proglottis à testicules et ovaire développés sont rectangulaires ou un peu trapézoïdaux, env. 2 fois plus larges que longs $(0,42$ de long sur 0,84 de large). Les proglottis plus âgés atteignent une largeur de $1 \mathrm{~mm}$., 4 pour la même longueur de 0,42 . Lorsque commencent à se former les capsules ovifères, les proglottis deviennent carrés, puis augmentent de longueur et diminuent de largeur; les gravides arrivent à être un peu plus de deux fois aussi longs que larges, par exemple $2 \mathrm{~mm} ., 4$ de long pour $1 \mathrm{~mm} ., 1$ de large (fig. 29).

Le nombre des testicules peut être évalué, très approximative- 
ment, à $30-35$; j'ai compté, par exemple, env. 12 à 14 poraux pour 20 antiporaux ; ils dépassent les gros canaux excréteurs et atteignent, ou presque, le nerf (fig. 28). La poche du cirre est loin d'atteindre le gros canal excréteur ; j'ai mesuré une taille minimum de $106 \times 48 \mu$; pour des proglottis sexués, j'ai trouvé env. $115 \times 55$; pour un proglottis sexué de 0,378 de long sur 0,840 de large, j'ai

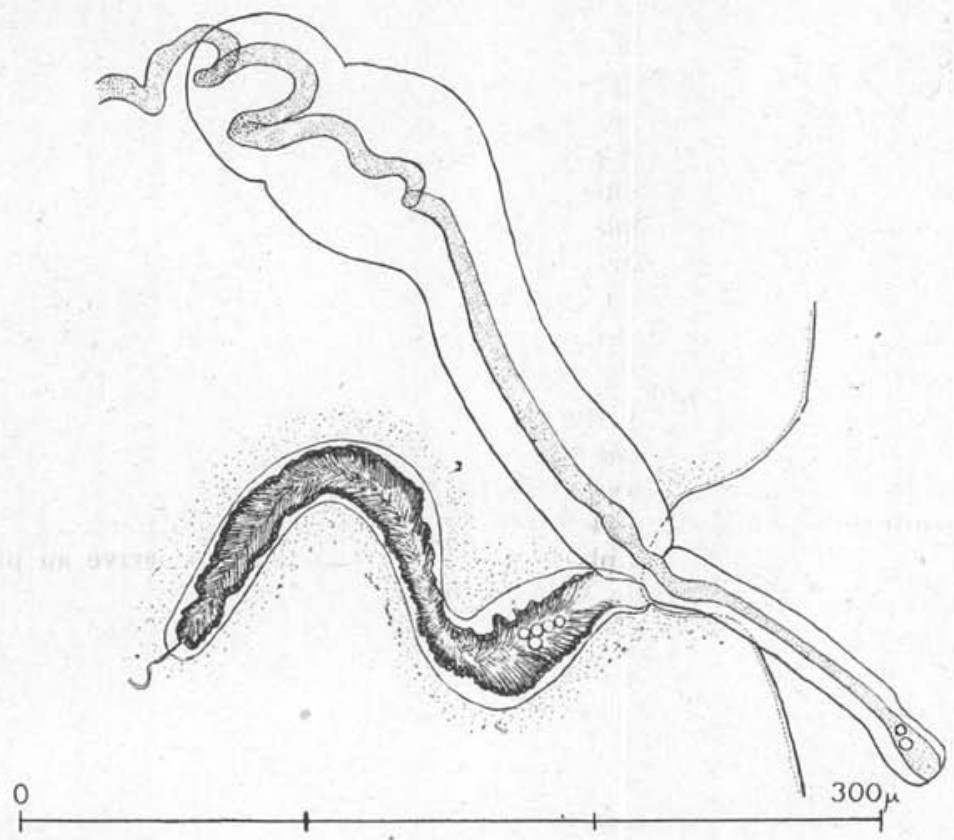

Fig. 23. - R. (R.) equatoriensis R.-Ph. Dollfus 1939. Poche du eirre et vagin. Le cirre fait saillie par le pore génital.

mesuré $136 \times 52 \mu$. La partie distale différenciée du vagin est incurvée en $\mathrm{U}$, le réceptacle séminal est mal distinct et paraît inconstant. Le pore génital est profond et accompagné d'un sphincter bien visible. Tous les pores sont du même côté, un peu en avant ou en arrière du premier tiers de la longueur du proglottis, mais toujours en avant de la moitié.

L'ovaire est une masse unique, brièvement lobée, large d'env. 0,22 , non déplacée vers le côté poral. Le vitellogène, apparemment non lobé, ne dépasse pas 0,12 de large.

Il y a env. 75 à 80 capsules ovifères, elles sont du type parenchymateux habituel (fig. 30), considéré par C. R. Lopez-Neyra 
comme caractéristique du genre Kotlania. Chacune renferme env. de 3 à 6 œufs de 23-26 $\mu$. Dans les proglottis gravides, le parenchyme cortical disparaît presque complètement et les capsules viennent au contact de la paroi du corps.

Le Raillietina des $\mathrm{D}^{\mathrm{r}}$ R. H. Loeches et P. Alavez ne correspond à aucune espèce connue de l'homme et des mammifères, je propose

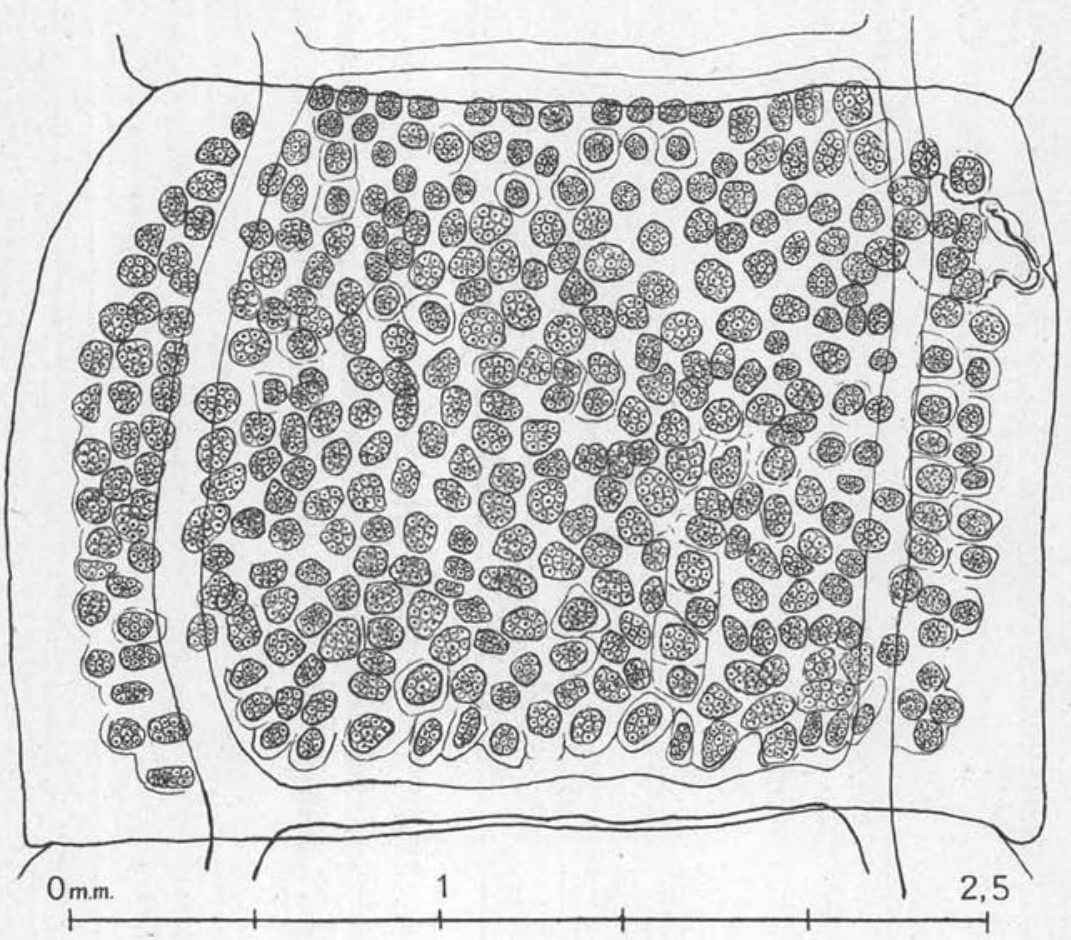

Fig. 24. $-R$. (R.) equatoriensis R.-Ph. Dollfus 1939. Proglottis à maturité.

de le nommer $R$. (R.) loechesalavezi n. sp., en l'honneur des praticiens qui ont pris la peine de le récolter et ont attiré l'attention sur l'existence de la raillietinose à Cuba, déjà en 1930 (1).

La nouvelle espèce se distingue facilement de $R$. demerariensis (C. W. Daniels) par un nombre de testicules moitié moindre, une poche du cirre sensiblement plus courte, son ovaire non bilobé et

(1) Les périodiques médicaux locaux, de Cuba, ne me sont pas accessibles, aussi ne puis-je pas savoir si l'observation des $\mathrm{D}^{\text {rs }} \mathrm{R}$. H. Loeches et P. Alavez a été publiée. 
plus petit, son cirre non épineux, ses capsules ovifères beaucoup moins nombreuses ; c'est cependant de demerariensis, tel qu'il a été redécrit par C. R. Lopez-Neyra, que loechesalavezi se rapproche le plus.

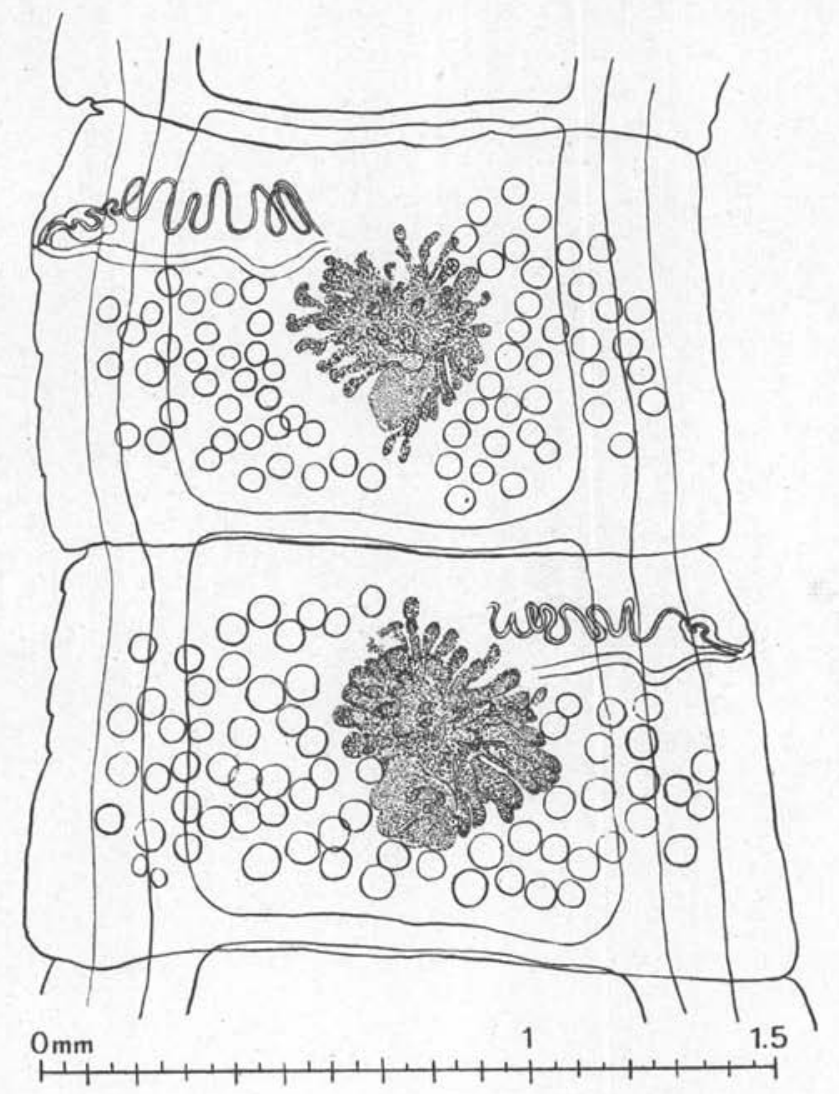

F1G. 25. - R. (Fuhrmannetta) leoni R.-Ph. Dollfus 1939. Proglottis sexués, pores alternes.

Comme autre information sur la raillietinose humaine à Cuba, nous avons, ainsi qu'il a été dit plus haut, la récente publication des $\mathrm{D}^{\text {rs }}$ Pedro Kouri et José Macho Doval ; elle est relative à trois cas, l'un chez un nourrisson de 18 mois en 1935, les autres chez des enfants en 1937. Les deux auteurs ont fait suivre l'histoire clinique d'une description du seul exemplaire complet du parasite qu'ils ont observé. 
Leur description (1938 a, p. 126-129, fig. 2-7 ; $1938 b$, p. 213-215, pl. XL-XLV, fig. 2-7) se ramène à ceci :

Longueur totale : $39 \mathrm{~cm}$. ; 248 proglottis à partir d'une distance de $22 \mathrm{~mm}$. du scolex. Pore génital unilatéral au milieu de la longueur de l'anneau. Poche du cirre petite. Environ 150 capsules ovifères contenant approximativement 3 à 6 œufs.

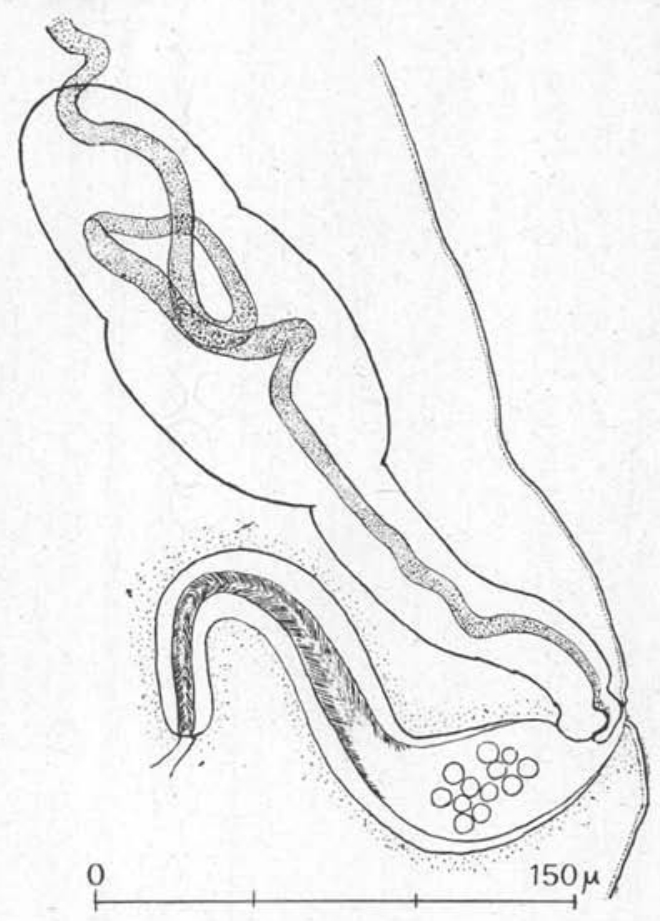

F1G. 26. - R. (F.) leoni R.-Ph. Dollfus 1939.

Poche du cirre et vagin. Autofécondation.

Au lieu de donner, de leurs préparations, des dessins à la chambre claire avec une échelle millimétrique permettant des mesures au compas, P. Kouri et J.-M. Doval ont accompagné leur description de photographies. Sur 31 photographies, trois représentent, en grandeur naturelle ou à un faible grossissement, le spécimen entier et des fragments du deuxième spécimen; les autres sont des microphotographies à des grossissements divers, non indiqués; pour quelques-unes il y a l'indication d'un numéro d'oculaire et d'un numéro d'objectif, ce qui n'apporte aucun renseignement utilisable sur les dimensions des organes. II 
aurait été important de pouvoir mesurer, sur les photographies, la largeur du scolex, le diamètre des ventouses, la longueur et la largeur

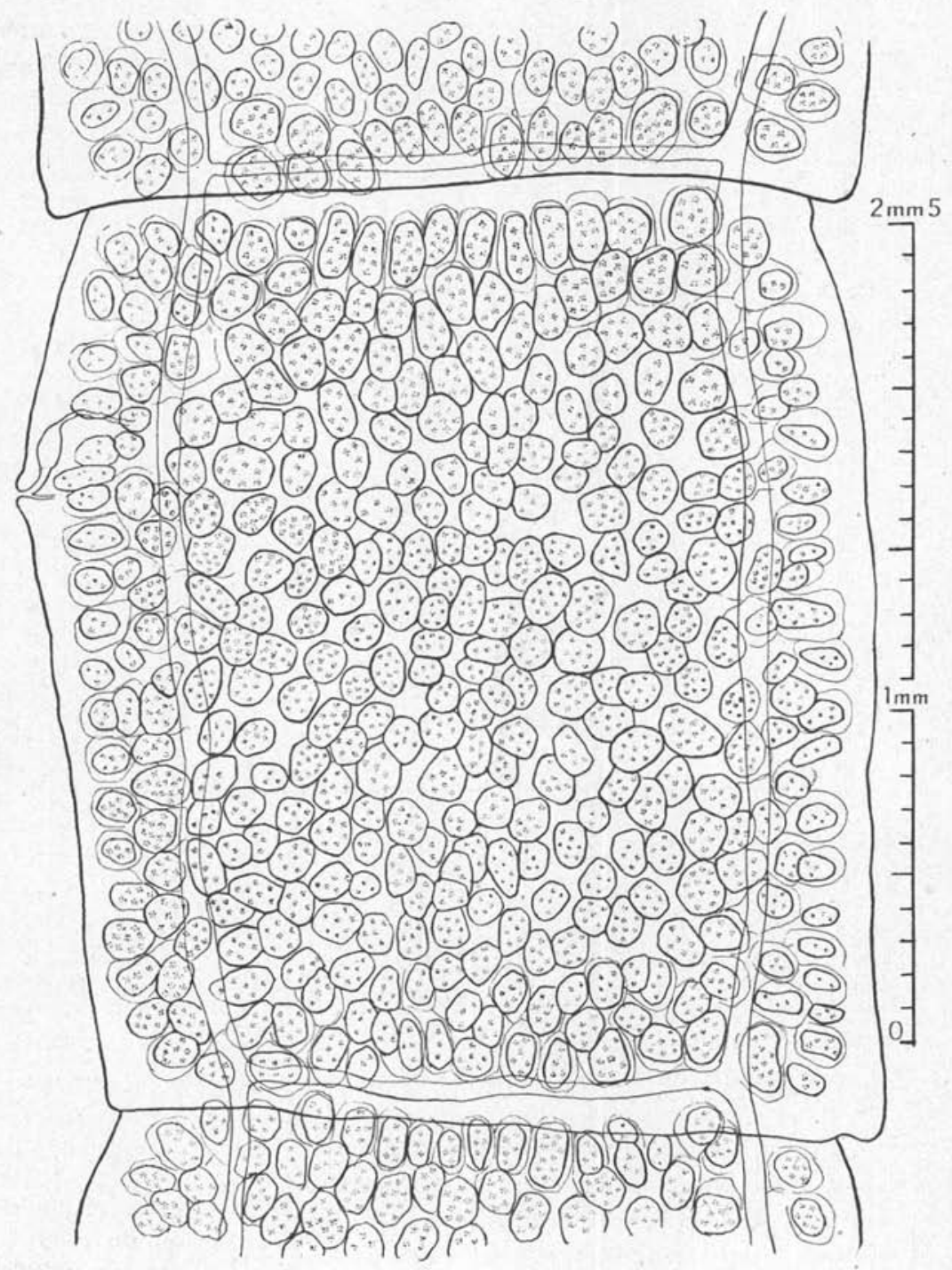

Fıc. 27. $-R$. (F.) leoni R.-Ph. Dollfus 1939. Proglottis à maturité.

des proglottis aux divers états de maturité, le diamètre des capsules ovifères, des œufs, etc..., mais aucune échelle ne le permet. 
Les photographies montrent néanmoins que les proglottis sont d'abord beaucoup plus larges que longs, que leur longueur augmente avec leur maturité sexuelle, qu'ils deviennent carrés puis plus longs que larges et que, gravides, ils arrivent à être un peu plus de deux fois aussi longs que larges; sur deux des photographies, j’ai compté environ 112 capsules ovifères polygonales, sur une autre environ 160 capsules.

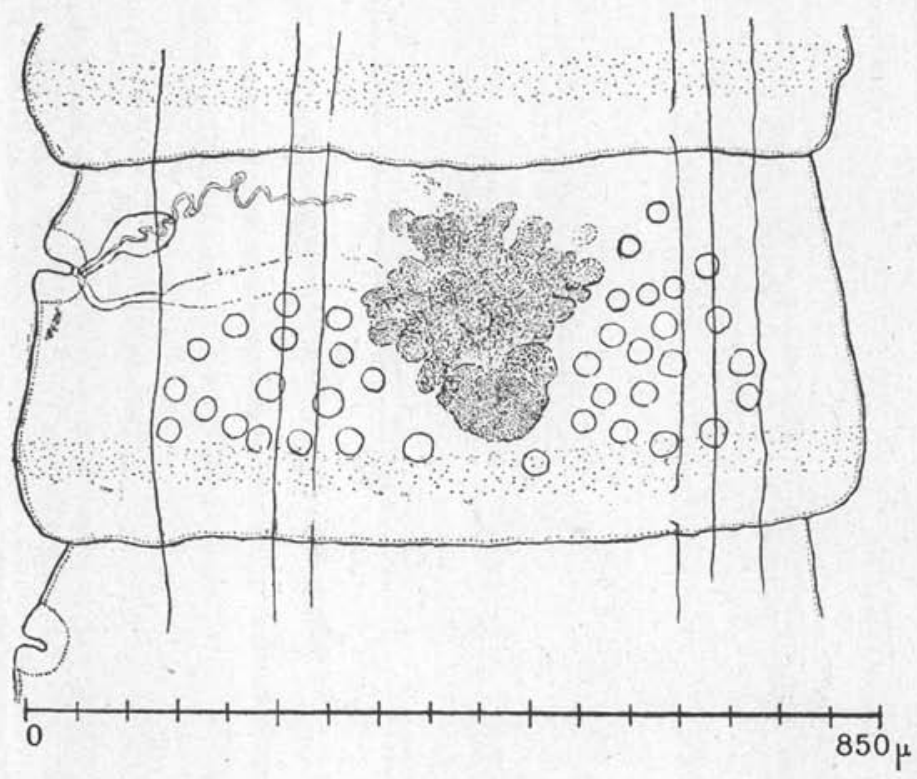

Fig. 28. - R. (R.) loechesalavezi n. sp. Proglottis sexué.

Il n'apparaît pas que le Raillietina de R. H. Loeches et P. Alavez appartienne à la même espèce que celui de P. Kourí et J. M. Doval et la position du pore génital nettement au milieu de la longueur du bord de l'anneau, empêche toute identification avec demerariensis à pore génital dans le tiers antérieur. On ne connaît pas, non plus chez les mammifères, de Raillietina auquel celui de P. Kouri et J. M. Doval soit assimilable, je propose donc de désigner, au moins provisoirement, cette espèce sous le nom de $R$. (R.) Kouridovali n. sp., en l'honneur des deux praticiens de Cuba qui en ont donné, les premiers, une description. 


\section{Discrimination des huit formes de Raillietina} TrouvéEs CHEZ L'Homme en Amérique

Les huit formes de Raillietina distinguées ici parmi celles trouvées chez l'homme en Equateur, en Guyane et à Cuba, sont-elles bien toutes spécifiquement indépendantes ? Peut-être s'agit-il seu-

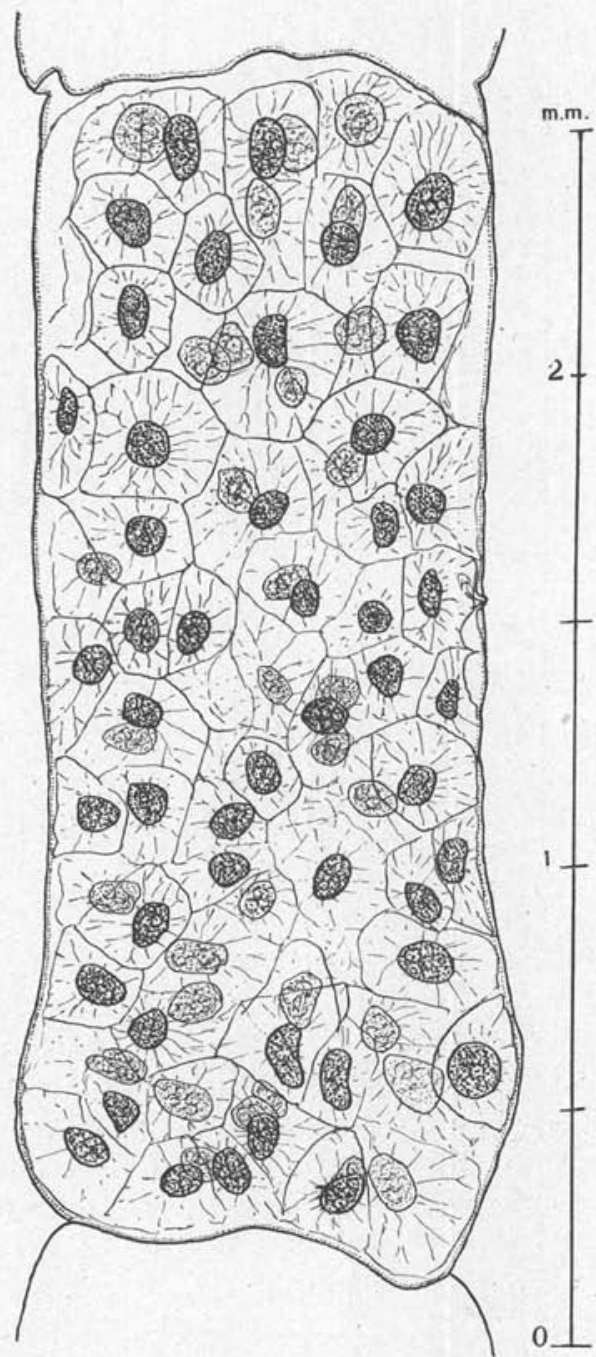

FIG. 29. - R. (R.) loechesalavezi n. sp. Proglottis gravide. 
lement de trois ou quatre espèces susceptibles de variations individuelles étendues? Je ne saurais me prononcer définitivement d'après le matériel que j'ai examiné, mais j'écarte toute identification avec les Raillietina connus en Asie et dans les îles indo-africaines chez l'homme et les mammifères, comme j'écarte toute iden-

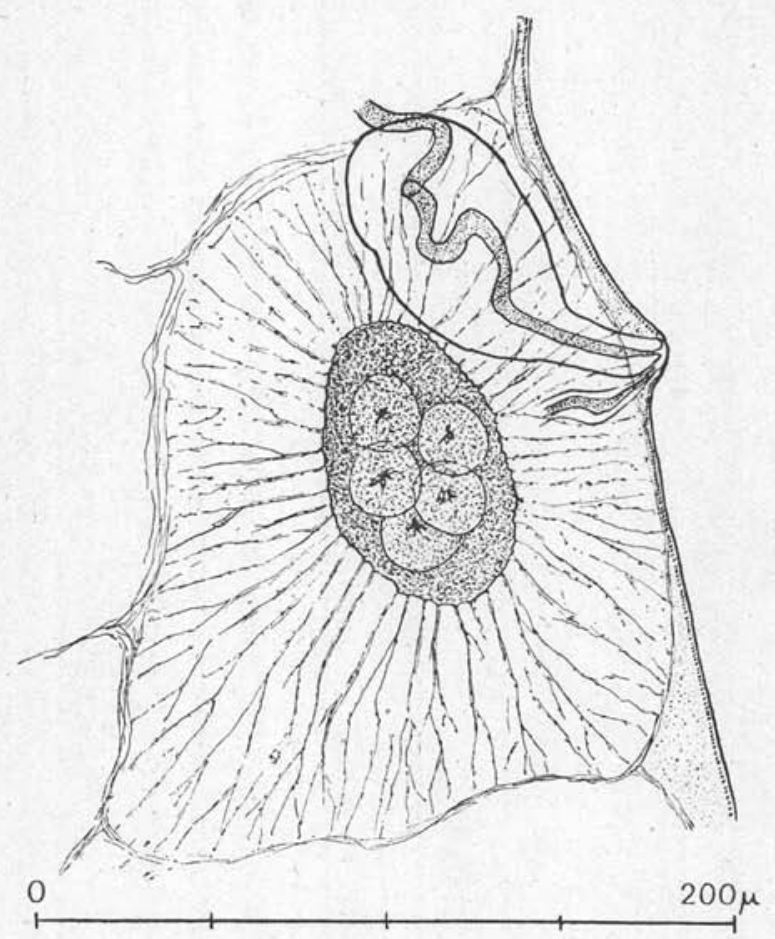

Fic. 30. -- R. (R.) loechesalavezi n. sp. Capsule ovifère située au niveau du pore génital.

tification avec les Raillietina d'Oiseaux. Il est évident que le jour où il sera démontré qu'une même espèce de Raillietina est susceptible de parasiter aussi bien un oiseau que l'homme ou un autre mammifère, une révision s'imposera de l'ensemble des espèces du genre.

Dans le tableau suivant, je présente une tentative pour aider à la discrimination des huit formes, variétés ou espèces de Raillietina néotropicales, que j'ai décrites ou dont j'ai rappelé les caractères. 
I. Espèces de petite taille (moins de $50 \mathrm{~cm}$.) (1)

A. Il y a production de proglottis gravides à une taille de moins de $20 \mathrm{~cm}$. Le pore génital est antérieur à la mi-longueur du proglottis sexué,

$\left.a_{1}\right)$ 75-80 capsules ovifères, cirre inerme
R. (Raillietina) lochesala- vezi, n. sp.

a $a_{2}$ 120-150 capsules ovifères, cirre

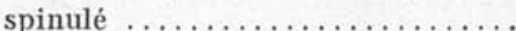

B. II y a production de proglottis gravides R. demerariensis (C.-W. Daniels). à une taille de $30-40 \mathrm{~cm}$. Le pore génital est à la mi-longueur du proglottis sexué,

110-160 capsules ovifères $\ldots \ldots \ldots \ldots$.

R. (R.) Kouridovali n. sp.

\section{Espéces de grande taille (de 1 à 10 mètres) (2)}

A. Proglottis devenant env, 2 fois plus longs' que larges à maturité, ovaire se séparant en deux groupes de lo-: bes en éven-

$a_{2}$ ) pores irrégulièrement alternes, ovai-

$\left.a_{1}\right)$ pores unilatéraux tail $\ldots \ldots \ldots$.

ovaire rassemblé en un seul massif lobé .. re en un seul groupe de lobes, plus ou moins rayonnants, ayant parfois tendance à se séparer en deux groupes $\ldots \ldots \ldots \ldots \ldots \ldots \ldots \ldots$

B. Proglottis ne devenant pas, ou à peine, plus longs que larges à maturité,

$b_{1}$ ) pores unilatéraux (avec irrégularités) vers le premier tiers de la lon-
R. (R.) equatoriensis R.-Ph. Dollfus.

R. (R.) quitensis, L.-A. León.

R. (Fuhrmannetta) leoni. R.-Ph. Dollfus.

(1) Ce tableau ne comprend pas le Raillietina de Luis G. Davila (1922), sur lequel je n'ai pas pu me documenter.

(2) La figure 1 de la pl. XLVI de la note de L.-A. León (1938) représente un individu de quitensis photographié en entier à côté d'une échelle centimétrique, ce qui permet d'admettre comme non exagérée une longueur d'au- moins 8 mètres. 
gueur du proglottis sexué, ovaire se séparant en deux groupes de lobes digités en éventail (1), capsules ovifères $140-180 \ldots \ldots \ldots \ldots$.

$b_{2}$ ) pores unilatéraux vers le premier R. (R.) brumpti, R.-Ph. Dollfus. quart de la longueur du proglottis sexué, ovaire se séparant en deux groupes brièvement lobés, non en éventail, capsules ovifères 170-220.

R. (R.) luisaleóni, R.-Ph. Dollfus.

\section{RÉSUMÉ}

Pour l'Amérique intertropicale, aucune espèce de Raillietina n'est connue chez des mammifères sauvages ou domestiques et, jusqu'à ces dernières années, ưne seule espèce, $(R$. demerariensis (C. W. Daniels 1895) de la Guyane anglaise, était connue de l'homme.

Depuis lors et en particulier de 1930 à 1938, plusieurs spécimens du genre ont été obtenus de l'homme en Equateur et à Cuba. Ayant examiné plusieurs d'entre eux, j'en donne la description et je sépare provisoirement, en huit formes qui ne sont peut-être pas toutes spécifiquement distinctes, l'ensemble des Raillietina néotropicaux de l'homme, qui me sont connus par des spécimens originaux ou des descriptions.

\section{BiBLIOGRAPHIE (2)}

BAER (J. G.). - A propos d'une nouvelle classification des Cestodes du genre Davainea R. Bl. s.l. Bull. Soc. Zool. France, LV, 1931, p. 44-57.

Blanchard (R.). - Tête de Davainea madagascariensis. Bull, Soc. Pathol. exot., IX, 1916, p. 413 .

(1) Pour connaitre la forme typique de l'ovaire chez un Raillietina, il ne faut pas se contenter d'examiner seulement trois ou quatre proglottis, il faut en examiner un grand nombre assez avancés dans leur développement; la séparation en deux groupes, des lobes ovariens, n'est généralement pas précoce.

Je suppose que l'on peut souvent tirer de l'habitus de l'ovaire un caractère utile pour la discrimination spécifique. L'ovaire est en deux masses séparées non lobées chez $R$. garrisoni M. A. Tubangui 1931 et chez $R$. murium Ch. Joyeux et J. G. Baer 1936 ; il est lobé en un éventail unique chez $R$. echinobothrida (Mégnin 1881), etc...

(2) Il ne me paraít pas utile de présenter iei une bibliographie étendue sur les Raillietina de l'homme et les mammifères : une telle bibliographie a déjà été publiée par J. G. Baer 1931, Ch. Joyeux et J. G. Baer 1929a, C. R. LopezNeyra 1931 ; je renvoie donc à ces ouvrages. Pour les références postérieures, on les trouvera dans les listes bibliographiques données dans ceux plus récents, parmi les ouvrages ci-dessous. Le signe * indique que je n'ai pas consulté l'ouvrage. 
Brumpt (E.). - Précis de parasitologie. Paris, Masson et Cie, $5^{e}$ édition, 1936, vol. I.

CHEN (H. T.). - A preliminary report on a survey of animal parasites of Canton, China, rats. Lingnan Science Journ., XII, 1933, p. 65-74.

Daniels (C. W.). - - Trenia demerariensis. British Guiana Med. Ann., Demerera, VII, 1895 , p. $95-98$, pl.

- Tania demerariensis (?). Lancet, London, $\mathrm{n}^{\circ} 3821,1896$, vol. II, p. 1455 , fig. 1-4.

Davila (L.-G.). - - [Communication au VI Congreso médico Latino Americano]. Anales de la Universidad Central, Quito, 1922, $\mathrm{n}^{\circ} 34$.

Dollfus (R.-Ph.). - Cestodes du genre Raillietina récemment observés chez l'homme en Equateur. Bull. Soc. Pathol. exot., XXXII, 1939, p. 660-665 et erratum, p. 960 .

HoNda (D.). - On a new Cestode, Raillietina (Raillietina) coreensis n. $\mathrm{sp}$. from a field mouse, Apodemus agrarius corex in Chosen. Jl. Chosen Med. Assoc., XXIX, 1939, p. 41-45, fig.

Hsü (H.-F.). - Contribution à l'étude des Cestodes de Chine. Revue suisse de Zool., XLII, 1935, p. 477-570, fig. 1-68.

JANıCK: (C. v.). - Zur Kenntnis einiger Säugetiercestoden. Vorläufige Mitteilung. Zool. Anzeiger, XXVII, 1904, p. 770-782.

Studien an Säugetiercestoden. Zeitschr. wiss. Zool., LXXXI, 1906, p. 505597, fig. texte 1-15, pl. XX-XXV, fig. 1-8.

Joyeux (Ch.) et BAER (J.-G.). - Etude de quelques cestodes provenant des colonies françaises d'Afrique et de Madagascar. Annales de paras., V, 1927, -p. 27-36, fig. 1-8.

- $\quad$ a. Les cestodes rares de l'Homme. Bull. Soc. Pathol. exot., XXII, 1929, p. 114-136.

b. Raillietina (Raillietina) celebensis Janicki, 1902 et Raillietina (Raillietina) baeri Meggitt et Subramanian, 1927, Bull. Soc. Pathol. exot., XXII, 1929, p. 675-677.

Helminthes des rats de Madagascar. Contribution à l'étude de Davainea madagascariensis (Dav., 1869). Bull. Soc. Pathol. exot., XXIX, 1936, p. 611-619, fig. 1-2.

Kouri (P.) et Doval (J.-M.). - La raillietinosis human en Cuba. Bol. mens, clinica Asociación Damas Cavadonga. La Habana, V, 1938, p. 121-134, fig. 1-7.

Tres casos de parasitismo humano por especies de la familia Davaineidx. Revista Med. Trop. Paras., La Habana, IV, 1938, p. 207-217, pl. XXIX-XL, fig. 1-7.

Tres casos de parasitismo humano por especies de la familia Davaineidx. Extracto (Seccion de Parasitologia del VII Congresso de la Asociación medica Pan Americana. Enero 21 de 1938, Habana, Cuba). Revista Med. trop. Paras., La Habana, V, 1939, p. 59-60.

León (Luis A.). - '....in Archivos de la Facultad de Ciencias Medicas, Quito, $\mathrm{N}^{\circ} 3,1933$.

Contribución al estudio de la parasitologia sudamericana. El género Raillietina y su frecuencia en el Ecuador. Revista Med. trop. Paras., La Habana, IV, 1938, p. 219-230, pl. XLVI-L, fig. 1-9.

Contribución al estudio de la parasitologia sudamericana. El género Raillietina y su frecuencia en el Ecuador. Extracto (Session de Parasitologia del VII Congresso de la Asociación medica Pan Americana. 
Enero 21 de 1938, Habana, Cuba), Revista Med. trop. Paras., La Habana, V, 1939 , p. 60.

Lopez Neyra (C. R.). - Relations du Davainea madagascariensis et des espèces parasites des mammifères. Considérations sur les Davainea. Annales paras., IX, 1931, p. 162-184, fig. 1:1-2:9.

Stiles (Ch. W.). - A revision of the adult tapeworms of hares and rabbits. Proceed. U. S. Nat. Museum, Washington, No 1105 , XIX, 1897, p. 145 235, pl. V-XXV. [Bibliography by Albert Hassall, p. 222-225].

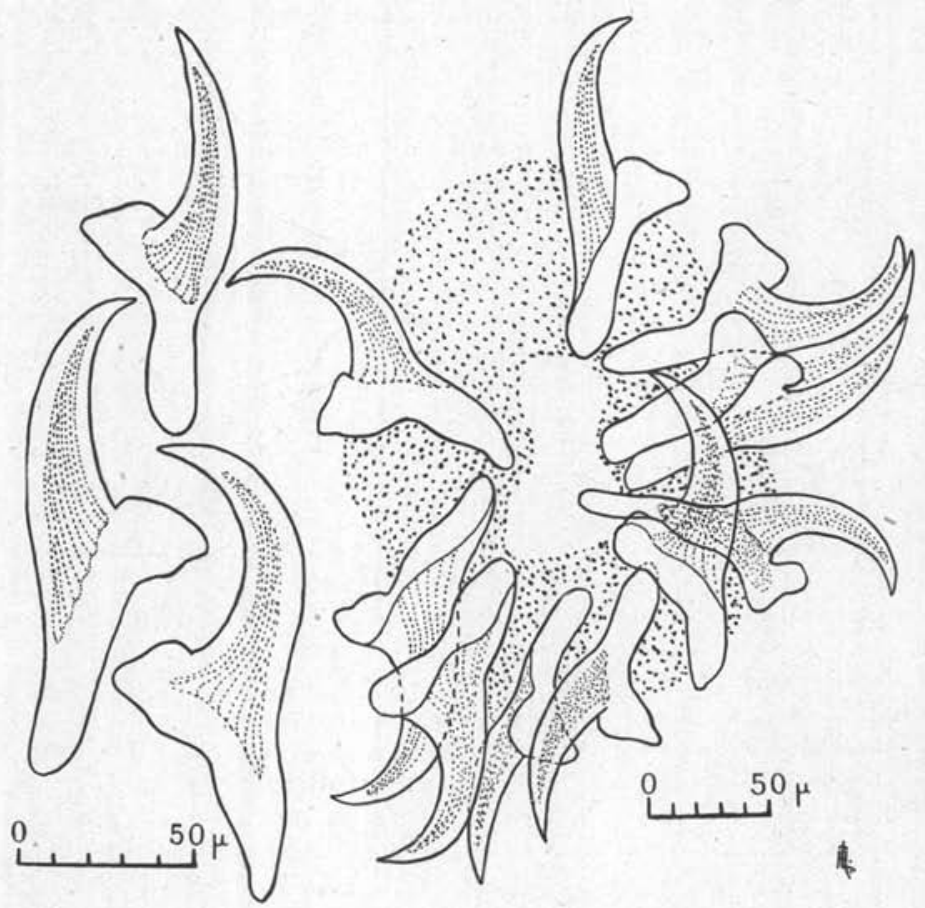

Fig. 31. - Trenia solium L. Crochets rostraux d'un specimen à pores irrégulièrement alternes.

\section{Note additionnelle}

Parmi les divers matériaux de parasitologie humaine en Equateur, contenus dans les derniers envois du $\mathrm{D}^{\mathrm{r}}$ Luis A. León au professeur E. Brumpt, se trouvaient deux préparations de scolex de Trnia et deux tubes de fragments de strobiles, indiqués comme Trenia, dont l'un correspondant au scolex de la seconde préparation. Je désigne ici les deux scolex comme $a$ et $b$ et le contenu des tubes comme $c$ et $d$.

a) L'étiquette du premier scolex porte seulement: «Trnia solium? 
Quito 1937 ». Le $D^{r}$ L.-A. León indique, dans une de ses lettres au professeur E. Brumpt, qu'il s'agit d'un Trenia dont la morphologie ne lui a pas paru caractéristique de solium, en particulier parce que les pores étaient très irrégulièrement alternes.

Le scolex a été fortement aplati; dans cet état il a une plus grande largeur de $0 \mathrm{~mm}$. 7 et une longueur approximative de $1 \mathrm{~mm} .5$; les ventouses ont un diamètre d'environ 0,225 à 0,246 . La ventouse apicale n'est pas mesurable. Il reste encore 11 crochets (fig. 31 ), ils sont de la même forme; la longueur de leur projection va de 106 à 123 et $127 \mu$ (1).

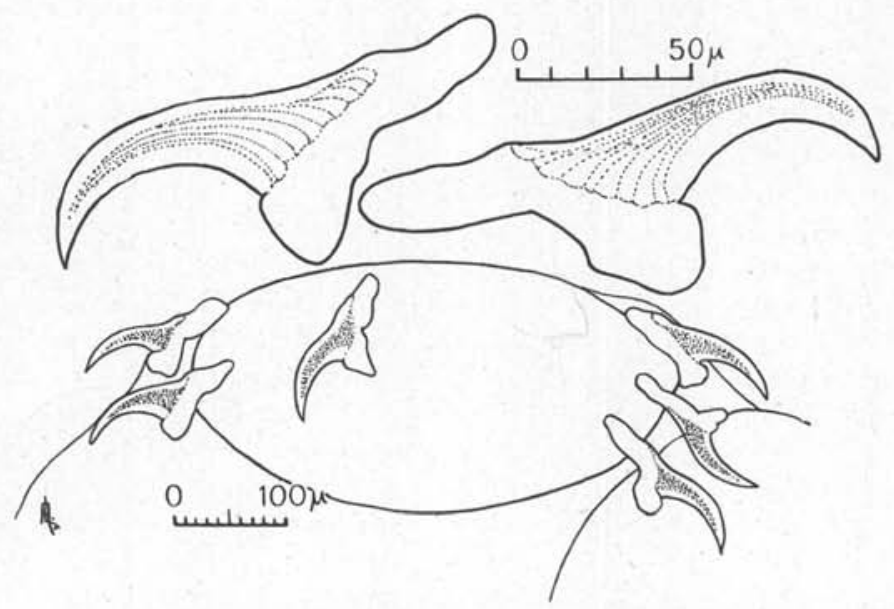

Fig. 32. - Trenia solium L. Crochets rostraux d'un specimen à pores irrégulièrement alternes.

Leur forme et leur taille sont compatibles avec celles des petits crochets de Trenia solium L., seule espèce de Tærnia armé dont la présence chez l'homme est connue. On peut supposer qu'il s'agit d'une espèce non encore signalée chez l'homme; mais, pour en décider, ce scolex, qui a perdu la plupart de ses crochets, n'est pas un matériel suffisant. J'estime donc, dans l'état actuel de nos connaissances, que ce scolex peut être provisoirement rapporté à solium.

b) L'étiquette de ce scolex porte seulement : «Tænia sp. Ecuador ». La préparation a été fortement aplatie ; dans cet état, la largeur du. scolex est d'environ $1 \mathrm{~mm}$. 3, les ventouses ont un diamètre de 0,42 (de 0,39 à 0,47 ); la ventouse apicale un diamètre d'environ 0,39 . Il ne reste plus que 6 crochets (fig. 32), dont la projection est longue de 139 à

(1) La comparaison avec un autre micromètre donne $110 \mu$ pour le moins long, $133 \mu$ pour le plus long. 
$143 \mu$ (1). La longueur du scolex, comptée de l'apex à la fin de l'élargissement (c'est-à-dire jusqu'où le ruban acquiert une largeur qu'il conservera égale sur plus d'un centimètre), est d'environ $1 \mathrm{~mm}$. 84 . La première indication perceptible de proglottisation se montre à environ $4 \mathrm{~mm} .38$ de l'apex du scolex.

La forme et la taille des crochets correspondant à ceux des petits crochets de solium et aucun caractère observable n'étant incompatible avec solinm, ce scolex me semble pouvoir être rapporté, au moins provisoirement, à solium.

c) Les fragments du strobile de ce tube correspondent, d'après les renseignements donnés par le $\mathrm{D}^{r}$ L.-A. León, au scolex de la préparation précédente. L’étiquette porte: «Tænia, intestino, mujer. Tumbaco (Canton Quito), Fecha IV-1934, Orden D. N-2. Le plus long fragment est long de $260 \mathrm{~mm}$. et comprend environ 539 proglottis, beaucoup sont carrés et mesurent environ 0,6 ; un autre fragment est long de $114 \mathrm{~mm}$. et comprend environ 203 proglottis plus larges que longs, par exemple : longueur 0,4 , largeur $0,7-0,8$, à long. 0,5 , largeur 1,4 ; les autres fragments sont plus courts, ne dépassant pas $50 \mathrm{~mm}$. de long, avec une largeur variable atteignant $5 \mathrm{~mm}$. Par exemple un fragment de $23 \mathrm{~mm}$. comporte 17 proglottis mesurant environ 0,135 de long sur $5 \mathrm{~mm}$. de large. Le tube contient en outre un certain nombre de proglottis gravides, par groupes de deux à six, ou même isolés, atteignant $11 \mathrm{~mm}$, de long sur 3 de large.

$c_{1}$ ) J'ai d'abord examiné les grands proglottis larges de 3 à $5 \mathrm{~mm}$.

Ils présentent l'anatomie habituelle des Trania du groupe solium. Chez un proglottis long de $3 \mathrm{~mm}$. 113, largè de $5 \mathrm{~mm}$. 235, assez âgé pour que l'utérus soit déjà bien formé, la poche du cirre, mesurée jusqu'au pore génital, est longue de $518 \mu$ (dont 224 pour sa partie proximale renflée) avec un plus grand diamètre de $140 \mu$, elle n'atteint pas tout à fait le gros canal excréteur ventral. Dans d'autres proglottis sexués, la longueur de la poche du cirre est à peu près la même : de 450 à $500 \mu$ pour un diamètre de 130 à $140 \mu$. Le pore génital est à peu près au milieu de la longueur du bord du proglottis, dans une importante dépression s'étendant sur une longueur de $0 \mathrm{~mm}$. 3 environ et une profondeur de $0 \mathrm{~mm}$. 1 environ. Les pores sont irrégulièrement alternes, il y en a souvent $3-4$ consécutifs du même côté. Dans les proglottis gravides, le pore est légèrement postérieur à la mi-longueur. L'utérus est très grêle, il montre, de chaque côté, 6 à 7 branches, se séparant souvent presque à angle droit; ces branches émettent en moyenne de 3 à 6 rameaux dont plusieurs peuvent être orientés longitudinalement (fig. 33) et se ramifier à leur tour. Les œufs sont sphériques, avec un diamètre d'environ 28 à $32 \mu$ et une coque épaisse d'environ $4 \mu$.

Si l'on excepte l'irrégularité de l'alternance des pores génitaux, rien ne paraît s'opposer à attribuer ces fragments sexués et gravides de Trenia, à solium. Il y a environ une quarantaine d'espèces valables dans

(1) La comparaison avec un autre mieromètre donne 138 à $147 \mu$. 
le genre Trenia (Voir les tableaux donnés, de leurs caractères comparés, par J.-G. Baer 1925, p. 51 et par R.-J. Ortlepp 1938, p. 275-276), mais on ne connait pas d'autre Tænia armé chez l'homme que solium; en existet-il une seconde en Amérique équatoriale? ce n'est pas impossible,

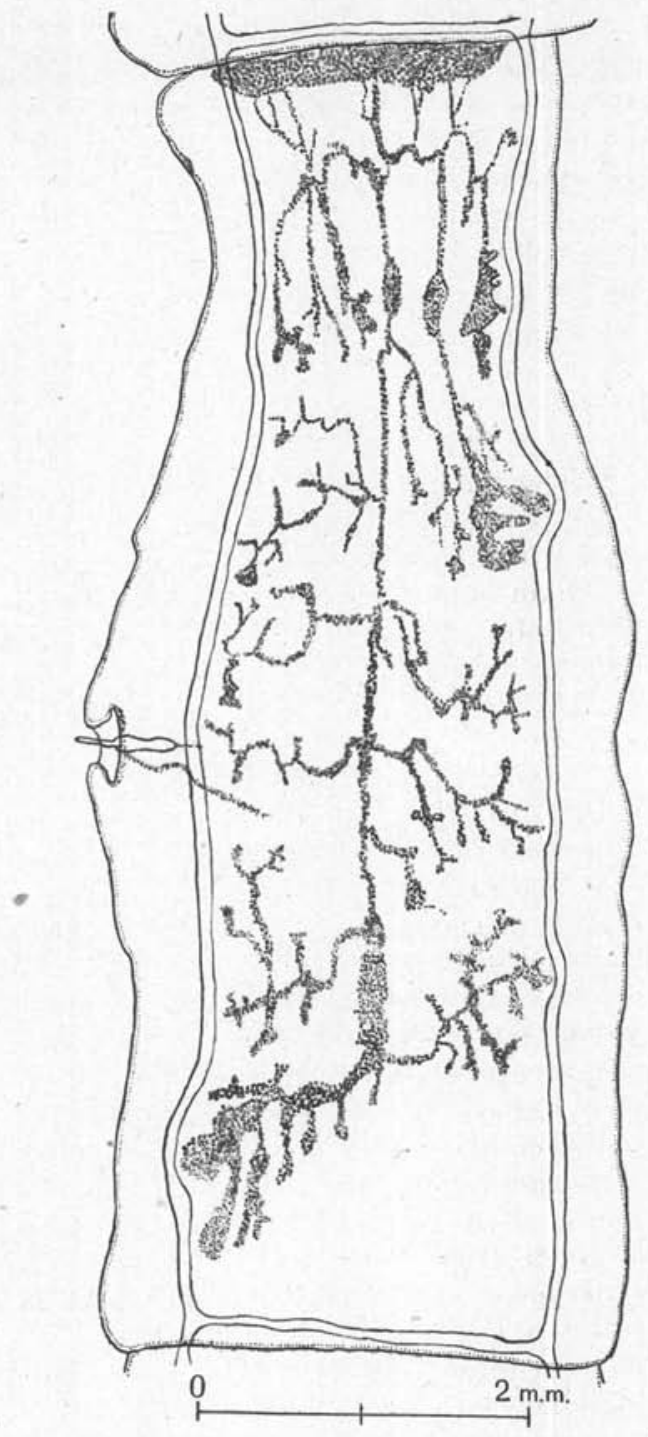

Fıc. 33. - Trenia solinm L. Proglottis gravide du même specimen que celui de la figure 32 . 
mais l'étude de spécimens entiers ayant leur scolex portant les crochets au complet, serait nécessaire pour l'établir manifestement. Remarquons que nous ne savons rien des espèces du genre Trenia parasitant les mammifères sauvages et domestiques de l'Equateur, ce qui nous prive de toute base pour supposer qu'une espèce de Trenia du groupe solium y est normalement parasite d'un mammifère et accidentellement de l'homme.

$c_{2}$ ) J'arrive maintenant à l'examen des autres fragments du même tube, fragments de strobile larges, au maximum, de $2 \mathrm{~mm}$. à $2 \mathrm{~mm}$. 5 .

A mon grand étonnement, j’ai constaté que, si plusieurs de ces fragments étaient formés de proglottis très jeunes, non sexués de Trnia, plusieurs autres avaient une anatomie complètement différente et étaient référables à un Raillietina du s. g. Fuhrmannetta.

Ceci conduit à admettre que le malade était parasité à la fois par un Trenia et un Raillietina et avait évacué deux vers qui, mélangés dans les selles avaient été regardés par L.-A. León comme des parties d'un seul.

Macroscopiquement, l'erreur est admissible et, en 1934, L.-A. León n'avait pas encore porté son attention sur les Raillietina.

Il s'agit d'un Raillietina à pores très irrégulièrement alternes, je constate, par exemple, 8 pores consécutifs d'un côté, puis 1 pore de l'autre, ensuite 14-1, 17-1, 16-1, 44-8, 18-2, 2-plus de 20. Le pore est au premier tiers dans les proglottis plus larges que longs, mais il arrive au premier quart dans les proglottis longitudinalement étirés comme celui de la figure 35 . La poche du cirre, dans les proglottis sexués, a, en moyenne $200 \times 60$ à $66 \mu$, elle n'atteint pas tout à fait le gros canal excréteur ventral. Le cirre est inerme, il y a souvent autofécondation. J'ai compté de 46 à 66 testicules (par exemple $22+24,24+27,25+33,26+40$, $27+38,28+32$ ) dont le diamètre atteint au maximum de 60 à $70 \mu$. Quelques testicules seulement dépassent parfois les gros canaux excréteurs ventraux.

L'ébauche de l'ovaire parait bilobée chez les proglottis qui ne sont pas encore tout à fait sexués (fig. 34), oủ la poche du cirre n'est pas complètement formée et représentée seulement par une ébauche. Lorsque l'ovaire a acquis son complet développement, il est fortement lobé presque digité et marque quelquefois une légère tendance à la séparation des lobes en deux groupes ; il mesure environ $0,30 \times 0,30$ à $0,30 \times 0,35$; son axe transversal est souvent oblique de même que celui du vitellogène.

Je ne puis que rapporter à $R$. $(F$.) leoni mihi, espèce très voisine de R. (R.) brumpti mihi, ces fragments de strobile. Leurs caractéristiques morphologiques ne coïncident pas complètement avec celles que j'ai indiquées dans ma description de leoni (Vide supra), mais c'était inévitable : il en est toujours ainsi lorsqu'une espèce a été décrite d'après un unique spécimen : l'étude des spécimens qui lui sont ensuite rapportés oblige à une révision de la diagnose pour qu'elle devienne applicable à l'ensem. ble des spécimens que l'on groupe sous le même nom spécifique.

d) Le tube contient un fragment de strobile de $73 \mathrm{~mm}$. de long sur 6 de 
large, comportant 39 proglottis. L'étiquette porte : "Tænia. Intestino. Mujer. Otavalo Imbabura. Fecha X-1935. Orden D. N.-3 \$. Ce matérie] semble n'avoir été fixé qu'après avoir subi un début de macération.

Les proglottis ont une longueur moyenne d'environ $2 \mathrm{~mm}$., ils sont

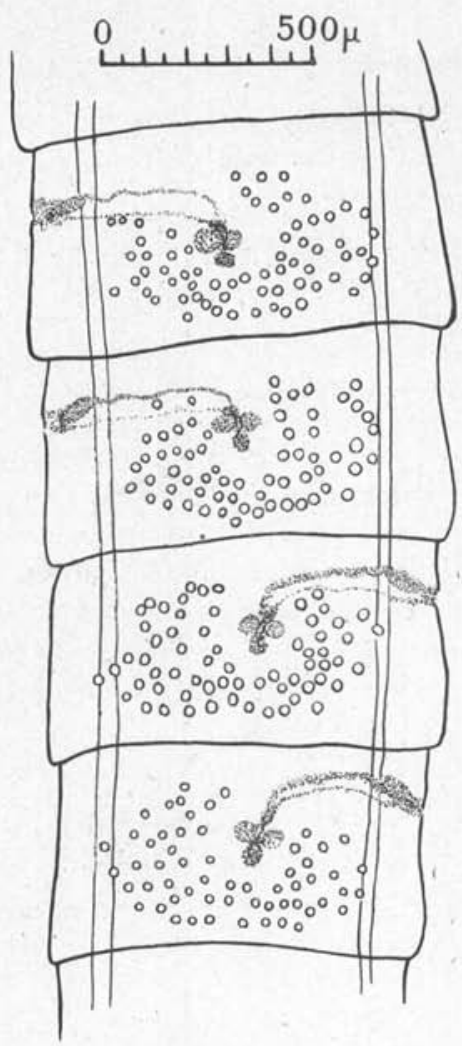

Fig. 34. - R. (F.) leoni R.-Ph. Dollfus 1939. Jeunes proglottis où la poche du cirre, le vagin, le canal déférent, l'ovaire sont encore à l'état d'ébauches.

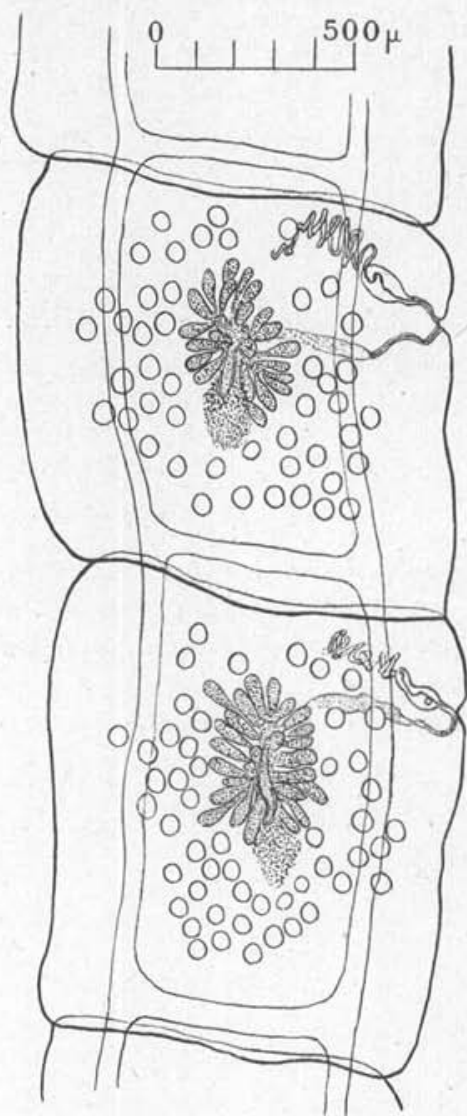

FIG. 35. $-R$. (F.) leoni R.-Ph. Dolifus 1939. Proglottis sexués d'une partie étirée du strobile.

sexués, mais non mûrs. La poche du cirre parait atteindre, ou presque, le bord externe du gros canal excréteur. Le pore génital a une ouverture circulaire avec un puissant sphincter. Les pores sont irrégulièrement alternes, il y en a jusqu'à 4 consécutifs du même côté. Comme il n'y a pas de proglottis mûrs, il n'est pas possible de connaitre la forme 
définitive de l'utérus gravide, mais l'utérus est assez avancé dans son développement pour montrer des branches latérales, elles sont au nombre de 3-4 de chaque côté. Il s'agit de la même espèce de Trnia que celle du tube $c$. Pour les raisons exposées plus haut, j'estime que, dans l'état actuel de nos connaissances, il ne peut être rapporté à une autre espère que solium.

\section{BIBLIOGRAPHIE}

BAER (J.-G.). - Contributions to the Helminth-Fauna of South Africa. 11 ${ }^{\text {th }}$ and $12^{\text {th }}$. Report Direct. Veter. Ed. and Research. Pretoria 1927, p. 63-136, 1 map, fig. 1-43.

Thèse Faculté des Sciences Univ. Neuchâtel, 1925, p. 1-79, 1 map, fig. 1-43.

Ortlepp (R.-J.). - South African Helminths. Part. II. Some Trenias from large wild carnivores. Onderstepoort JI. Veter. Sc., X, 1938, p. 253-278, fig. texte 1-19, pl. I, fig. 1-11.

Laboratoire des Productions coloniales d'origine animale du Muséum National d'Histoire naturelle de Paris et Institut de Parasitologie de la Faculté de Médecine de Paris 